\title{
Risk stratification by regadenoson stress MRI in patients with known or suspected coronary artery disease
}

\author{
Siddique A Abbasi ${ }^{1 *}$, Ravi V Shah²,1, Tomas Neilann, ${ }^{2,}$ Bobak Heydari', Hoshang Farhad ${ }^{1}$, Ron Blankstein ${ }^{1}$, \\ Michael Steigner ${ }^{1}$, Michael Jerosch-Herold ${ }^{1}$, Raymond $Y$ Kwong $^{1}$
}

From 17th Annual SCMR Scientific Sessions

New Orleans, LA, USA. 16-19 January 2014

\section{Background}

With the expanding use of vasodilator stress CMR, there has been a parallel rise in the use of regadenoson as a pharmacological stress agent. To date, however, prognostic data for stress CMR has focused on the use of adenosine or dipyridamole while prognostic data regarding the use of regadenoson is limited. We therefore sought to identify the prognostic value of regadenoson vasodilator stress CMR on clinical events in patients with known or suspected coronary artery disease.

\section{Methods}

We prospectively studied 346 consecutive patients clinically referred for CMR assessment of myocardial viability/ischemia over the course of 36 months. The CMR protocol consisted of: (1) cine SSFP functional imaging in multiple imaging planes, (2) stress perfusion imaging after administration of Regadenoson and a bolus injection of $0.1 \mathrm{mmol} / \mathrm{kg}$ intravenous gadolinium-DPTA; (3) late gadolinium enhancement imaging. Clinical follow-up was obtained via mailed questionnaire, review of electronic medical records, contact with the patients' cardiologist, and/or telephone contact with the patients. We used a primary composite endpoint of cardiovascular death or acute myocardial infarction.

\section{Results}

A total of 346 patients were prospectively followed for a median time of 1.9 years. There were 7 cardiac deaths and 8 acute myocardial infarctions during the follow-up period. By univariable Cox regression modeling, the presence of inducible ischemia was associated with a near-7fold increased risk of the primary outcome $(H R=6.95, P$ $=0.02$ ). Patients with absence of inducible ischemia experienced a low annualized cardiac event rate of $0.6 \%$ per patient year. To the contrary, patients with inducible ischemia had an annualized rate of cardiac event of 3.2\% per patient year.

Table 1 Univariable associations for cardiovascular death or acute myocardial infarction

\begin{tabular}{|c|c|}
\hline \multicolumn{2}{|c|}{ Univariable Associations } \\
\hline Characteristic & Hazard Ratio $(95 \% \mathrm{Cl})$ LR $\chi 2$ P-value \\
\hline Age & $1.01(0.96-1.07) 0.250 .62$ \\
\hline Female & $0.61(0.12-3.13) 0.360 .56$ \\
\hline $\mathrm{BMI}$ & $0.92(0.80-1.06) 1.320 .25$ \\
\hline History of HTN & $1.94(0.35-10.6) 0.590 .44$ \\
\hline History of DM & $2.16(0.42-11.2) 0.850 .35$ \\
\hline History of Smoking & $6.88(1.54-30.8) 6.370 .01$ \\
\hline History of Dyslipidemia & $0.70(0.13-3.81) 0.170 .68$ \\
\hline LVEF & $0.96(0.93-1.00) 3.590 .06$ \\
\hline LVEDVI & $1.01(0.99-1.02) 0.310 .58$ \\
\hline LVESVI & $1.01(0.99-1.02) 0.980 .32$ \\
\hline Presence of LGE & $8.59(1.03-70.9) 3.940 .04$ \\
\hline Inducible Ischemia & $6.95(1.35-35.8) 5.360 .02$ \\
\hline
\end{tabular}

Abbreviations: $\mathrm{BMI}=$ body-mass index, $\mathrm{HTN}=$ hypertension, $\mathrm{DM}=$ diabetes mellitus, LVEF = left ventricular ejection fraction, LVEDVI = left ventricular end diastolic volume index, LVESVI = left ventricular end systolic volume index, $\mathrm{LGE}=$ late gadolinium enhancement, $\mathrm{LR}=$ likelihood ratio.

'Brigham and Women's Hospital, Boston, Massachusetts, USA

Full list of author information is available at the end of the article 


\section{Conclusions}

Regadenoson stress CMR is a prognostically useful examination in which a negative study is linked to a low risk of adverse cardiovascular outcomes in a prospective cohort of 346 patients followed for nearly $1 \frac{1}{2}$ years. In addition, a positive regadenoson stress CMR is associated with a significantly higher annualized rate of adverse cardiovascular events in patients referred for known or suspected CAD, even after adjustment for clinical and demographic characteristics.

\section{Funding}

Astellas Pharma US, Inc.

\section{Authors' details}

'Brigham and Women's Hospital, Boston, Massachusetts, USA. ${ }^{2}$ Massachusetts General Hospital, Boston, Massachusetts, USA.

Published: 16 January 2014

doi:10.1186/1532-429X-16-S1-P186

Cite this article as: Abbasi et al:: Risk stratification by regadenoson

stress MRI in patients with known or suspected coronary artery disease.

Journal of Cardiovascular Magnetic Resonance 2014 16(Suppl 1):P186.

Submit your next manuscript to BioMed Central and take full advantage of:

- Convenient online submission

- Thorough peer review

- No space constraints or color figure charges

- Immediate publication on acceptance

- Inclusion in PubMed, CAS, Scopus and Google Scholar

- Research which is freely available for redistribution

Submit your manuscript at www.biomedcentral.com/submit
C Biomed Central 